Technical Note

\title{
Interpretability in Convolutional Neural Networks for Building Damage Classification in Satellite Imagery
}

\author{
Thomas Y. Chen ${ }^{1}$ (D) \\ 1 The Academy for Mathematics, Science, and Engineering, Rockaway, NJ 07866; \\ thomasyutaochen@gmail.com \\ * Correspondence: thomasyutaochen@gmail.com; Tel.: +1-973-295-8607
}

\begin{abstract}
Natural disasters ravage the world's cities, valleys, and shores on a monthly basis. Having precise and efficient mechanisms for assessing infrastructure damage is essential to channel resources and minimize the loss of life. Using a dataset that includes labeled pre- and post- disaster satellite imagery, we train multiple convolutional neural networks to assess building damage on a per-building basis. In order to investigate how to best classify building damage, we present a highly interpretable deep-learning methodology that seeks to explicitly convey the most useful information required to train an accurate classification model. We also delve into which loss functions best optimize these models. Our findings include that ordinal-cross entropy loss is the most optimal loss function to use and that including the type of disaster that caused the damage in combination with a pre- and post-disaster image best predicts the level of damage caused. Our research seeks to computationally contribute to aiding in this ongoing and growing humanitarian crisis, heightened by climate change.
\end{abstract}

Keywords: climate; disasters; interpretability; relief; satellite imagery

\section{Introduction}

Natural disasters devastate countless vulnerable communities and countries every decade. They are responsible for the deaths of 60,000 people a year worldwide, on average [1]. The timely allocation of resources in the event of these tragedies are crucial to saving lives. Additionally, natural disasters cause varying levels of damage to buildings. The havoc wreaked by them causes widespread infrastructure damage, in some cases leading to a "cascade effect" [2]. The resulting economic impact is colossal. For example, since 1980, the United States has sustained 273 weather and climate disasters that have caused damages exceeding 1 billion US dollars (USD), totalling 1.79 trillion USD [3]. Unfortunately, the frequency and severity of these disasters will only continue to increase, exacerbated by climate change [4]. The catastrophic impact of natural disasters and their increasing prevalence motivates the problem addressed in this work.

In order to prepare for and recover from these terrible but inevitable events, robust emergency response plans must be in place. This is especially needed in a world where weather and climate disasters are increasing in occurrence and brutality. This requires quickly and accurately analyzed data from the disaster site. Because it is almost always slow and hard (and sometimes impossible) to obtain damage assessment and other details from on the ground in a timely manner, satellite imagery has gained popularity in being used to analyze these types of situations. Deep neural networks (DNNs), which are machine learning algorithms that have multiple layers, have been used to locate and classify building damage within satellite imagery [5-8]. However, the current literature (see Literature Review) is limited in the interpretability of what exactly these neural networks are learning and what is most useful in assessing building damage. 
To better address this problem, we present a novel analysis of the most important information that a deep learning model needs to assess building damage. We use a convolutional neural network (CNN) architecture called a residual neural network (ResNet), pre-trained on Imagenet data [9]. In our approach, we train multiple CNNs on xBD satelite imagery data [10], with different modalities of input, as well as using different loss functions, and compare accuracy on the validation set. We aim to explicitly provide insight into the most effective ways to train models to classify levels of building damage, maximizing the efficiency of the emergency response after a natural disaster, which has the potential to save lives and reduce economic strain. We emphasize interpretability and reproducibility, particularly for the benefit of the end-operators of this technology.

\subsection{Literature Review}

\subsubsection{Image Classification}

Image classification is a well-studied area with work ranging from classical techniques [1114] to deep learning-based techniques [15-17]. Such deep-learning techniques have been enabled by ImageNet data [18]. Classification enables many opportunities in applications, such as object classification [19], scene classification [20], and action recognition [21]. In this work, we adapt the common image classification problem to damage assessment of buildings, using labeled satellite imagery.

\subsubsection{Computer Vision for Satellite Imagery}

Satellite imagery is useful in a plethora of areas, including for assessment of marine ecology [22], weather forecasting [23], and even in studying and predicting the spread of infectious disease [24]. Computer vision is the study of how computers gain high-level understanding from digital images and video. Used in tandem with satellite imagery, there are many possibilities for applications. Lately, there has been increased interest in using satellite imagery for humanitarian purposes such as responding to natural disasters [25]. Satellite imagery also provides insights for agriculture [26] and urban road damage [27]. More generally, change detection, which is the process of identifying differences in the state of an object by observing it at different times, can be used with satellite imagery in a variety of contexts. There are a few primary categories in which change detection approaches fall: algebra-based, transform-based, and classification-based [28]. Change detection has been employed on satellite imagery to study deforestation [29], urban growth [30], and more. In our case, the change between the pre-disaster image and post-disaster image can help in assessing and classifying building damage.

\subsubsection{Building Damage Assessment}

One specific area that has garnered significant attention in computer vision and satellite imagery is building damage assessment. Recent works have studied semantic building segmentation $[5,6]$ and cross-region transfer learning to avoid overfitting [7]. Furthermore, Gueguen and Hamid present a semi-supervised approach [8]. xView2 recently introduces a dataset, $x B D$, discussed more in detail later in this work [10]. Many teams [31] competed in the xView2 data competition and improved on the baseline model provided [10]. In our work, we focus on building damage assessment via image classification and change detection. We specifically hone in on what information is most useful in accurate classifications of building damage and analyze which loss functions are most fit for training our models and yield the most precise results. Our primary contribution is to improve upon the interpretability of machine learning models of prior works and existing literature in this area by explicitly examining per-building classification prediction accuracy with different combinations of inputted information and loss functions. 


\section{Materials and Methods}

\subsection{Dataset Details}

For this project, we utilize the xBD dataset [10], which consists of 22,068 high-resolution, annotated 1024 by 1024 satellite images for the purpose of building damage detection. This dataset covers a wide range of disasters in fifteen countries around the world (Fig. 2), from Guatemala to Portugal to Indonesia (over 850,736 building polygons totalling an area of 45,361 square kilometers). One of XBD's main purposes is to demonstrate changes between pre-disaster and post-disaster satellite imagery to aid in detecting the damage caused. Therefore, each post-disaster building is labeled as one of the following: "unclassified," "no damage," "minor damage," "major damage," or "destroyed." (We later discard the "unclassified" buildings, as described later in this section). The classification technique utilized is called the Joint Damage Scale (JDS), as presented and described in detail in Figure 1. We use the $\times B D$ dataset because it incorporates a variety of disaster types, building types, and geographical locations. This allows for diversity in training the model. For example, the wide variety of geographical locations is important for cross-region generalization. Additionally, the high resolution imagery allows for detailed change detection between pre-disaster and post-disaster images. These factors currently make xBD the leading dataset for building damage detection using labeled satellite imagery [10]. Previous satellite imagery datasets were not as comprehensive, and, for example, had only covered singular disaster types or did not have uniform building damage assessment criteria like the JDS used in XBD [32-34].

\begin{tabular}{|c|l|}
\hline Score Label & Visual Description of the Structure \\
\hline 0 No damage & $\begin{array}{l}\text { Undisturbed. No sign of water, structural damage, } \\
\text { shingle damage, or burn marks. }\end{array}$ \\
\hline 1 Minor damage & $\begin{array}{l}\text { Building partially burnt, water surrounding the } \\
\text { structure, volcanic flow nearby, roof elements } \\
\text { missing, or visible cracks. }\end{array}$ \\
\hline 2 Major damage & $\begin{array}{l}\text { Partial wall or roof collapse, encroaching volcanic } \\
\text { flow, or the structure is surrounded by water or } \\
\text { mud. }\end{array}$ \\
\hline
\end{tabular}

Figure 1. Joint Damage Scale descriptions, which are used in the labeling of the xBD dataset. Table taken from $x B D$ dataset release ${ }^{1}[10]$.

\subsection{Data Preprocessing}

We preprocess the data and train CNNs. The dataset consists of 1024 pixels by 1024 pixels satellite images. In order to zero in on the changes we begin by collecting bounding boxes of the buildings in each image. As aforementioned, the dataset provides segmentation ground truth masks with classification labels for each corresponding building polygon, which is a collection of Cartesian coordinates. Thus, we take the maximum and minimum $x$ coordinates of each polygon, $x_{\max }$ and $x_{\min }$, respectively, and the maximum and minimum $y$ coordinates, $y_{\max }$ and $y_{\min }$, respectively, and construct a bounding box with a lower left corner at coordinates $\left(x_{\min }, y_{\min }\right)$, width $x_{\max }-x_{\min }$, and height $y_{\max }-y_{\min }$. A visual representation of bounding boxes is displayed in Figure 3. 


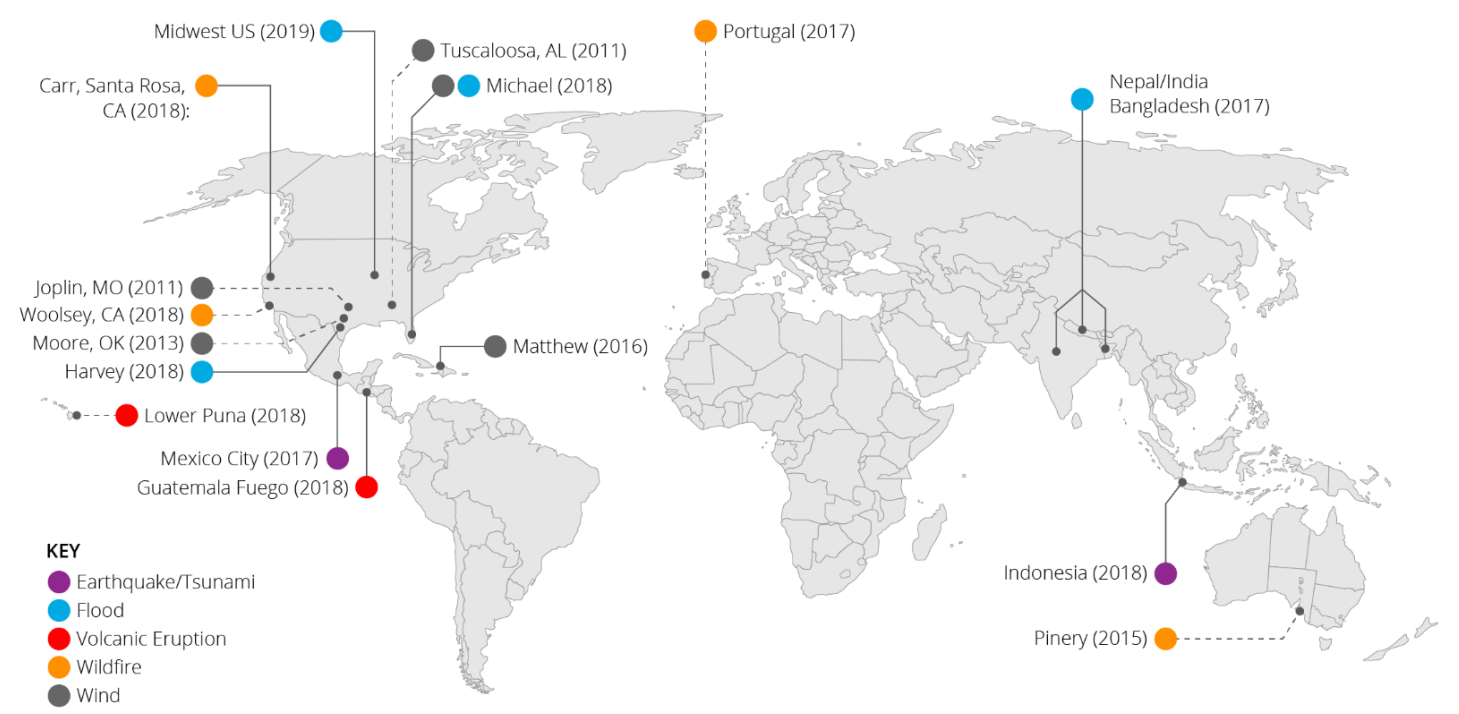

Figure 2. Map of locations worldwide from which satellite imagery data was taken. Figure taken from xBD dataset release ${ }^{2}[10]$.

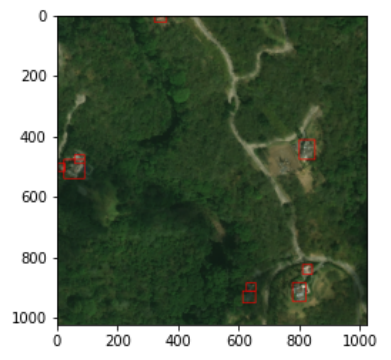

(a) Bounding boxes on an image

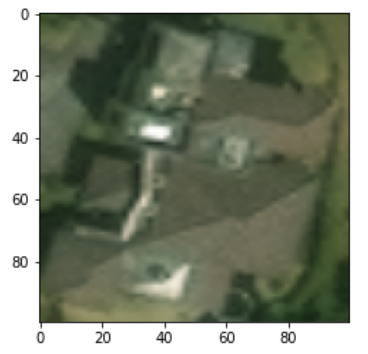

(b) One such bounding box and constituents

Figure 3. Left: Nine bounding boxes drawn in red on an arbitrary image from the dataset, each containing a building. Right: A building crop (the interior of one of these bounding boxes), resized to 100 pixels by 100 pixels.
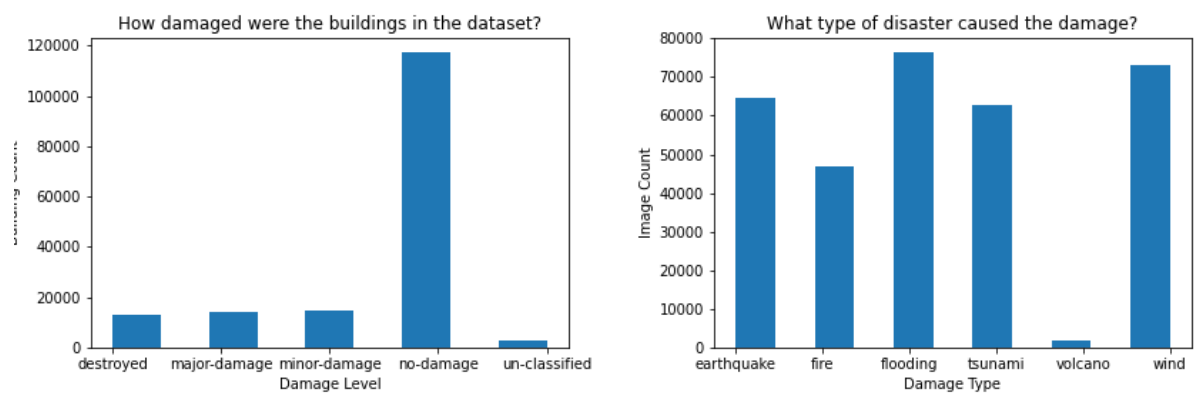

Figure 4. Left: Distribution of buildings by damage level. Right: Distribution of buildings by damage type.

We create graphical representations of the data that we choose to utilize from the dataset, plotting the number of buildings per disaster type and per damage classification (Fig. 4). Furthermore, we analyze the distribution of building bounding box sizes (Fig. 5), noting that buildings that are too small and blurred will not be valuable training data and may hinder the model from achieving accurate results (Fig. 6). We discard buildings that have a bounding box size of less than 2,000 pixels. We also discard any buildings with the classification ground truth label of "unclassified," because 
this information is not useful for our purposes. We could then randomly shuffle the dataset and split it into training and validation sets according the ratio 0.8:0.2. However, in order to maintain an equal distribution over JDS classification (damage level) in our training and validation sets so that we can properly assess model accuracy, we provide for an equal number of buildings of the categories "destroyed," "major damage," "minor damage," and "no damage" in each set, while still maintaining a 0.8:0.2 ratio between train and validation. The xBD dataset is deliberately created with a disproportionately large volume of buildings with no damage (see Figure 4) [10], but training on such a lopsided data distribution would yield artificially high accuracy numbers and not give valuable results.

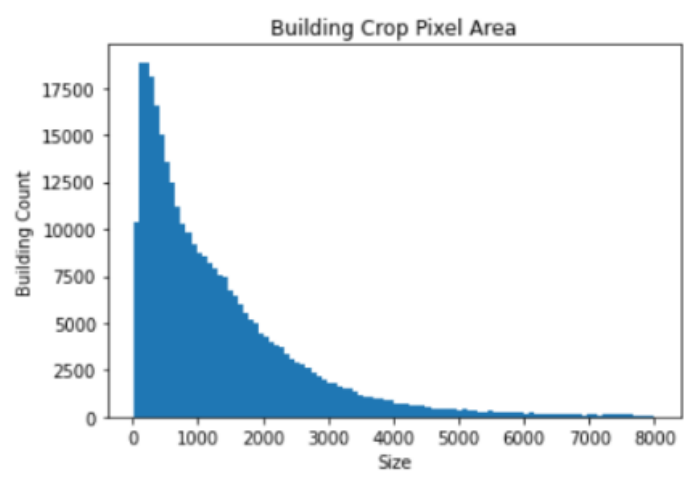

Figure 5. A histogram representing the distribution of building bounding box areas in the xBD dataset. Outliers (bounding boxes with a pixel area greater than 8000 ) have been removed from the graph.

64

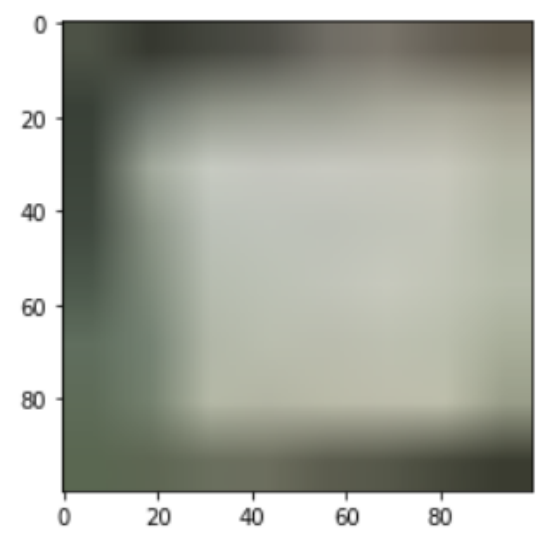

(a) 64 pixels.
6232

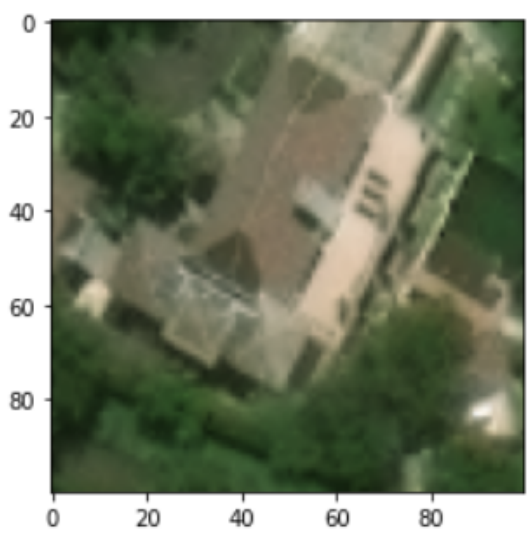

(b) 6232 pixels.

Figure 6. A comparison of two vastly differently sized buildings in the dataset. Both are resized to 100 by 100 pixels for viewing. Clearly, the blurred building of size 64 pixels would not be a useful data point for any deep learning model to learn from and yield accurate results. Instead, buildings like this are noisy and are discarded in our method.

\subsection{Baseline Model}

We train a baseline classification model to classify buildings by damage level, as defined by the Joint Damage Scale (Fig. 1). The model input is only the post-disaster image. Notably, our baseline model does not use change detection. Because the data is labeled, this is a supervised approach. The 
model architecture is ResNet18, an 18 layer CNN, and was pre-trained on ImageNet data [18]. This baseline model uses the cross-entropy loss function, which is defined as

$$
-\sum_{c=1}^{4} y_{o, c} \log \left(p_{o, c}\right),
$$

where $y_{o, c}$ is a binary indicator (either 0 or 1 ) of whether $c$, as a label, correctly classifies observation $o$, and $p_{o, c}$ is the predicted probability that observation $o$ is of the class $c$. Cross-entropy loss is defined, in other words, as the negative sum of the expression $y_{0, c} \log \left(p_{o, c}\right)$ across all 4 possible classes $c$ : no damage, minor damage, major damage, and destroyed. The network is trained on 12,800 buildings crops with a batch size of 32. The Adam optimizer with a learning rate of 0.001 is used. The model trained for 100 epochs on NVIDIA Tesla K80 GPUs. We use PyTorch [35] to train this model, as well as any subsequent models.

\subsection{Improvements}

We train other models that improve upon the performance of the baseline model. To do this, we introduce other model inputs, namely the pre-disaster image (in combination with the post-disaster image) and the type of disaster (e.g. volcano, wind, etc.) that caused the building damage. To train a model that takes in both pre-disaster images and their corresponding post-disaster images, we concatenate the RGB channels of the two and use that as input. To train a model that takes in the pre-disaster image, post-disaster image, and disaster type, we do the same, but also concatenate a one-hot encoded representation of the disaster type in one of the later layers of the CNN.

Furthermore, we experiment with other loss functions, namely mean squared error loss and ordinal cross-entropy loss to train these models. We define mean squared error as

$$
\frac{1}{b} \sum_{i=1}^{b}(y-\hat{y})^{2},
$$

where $b$ is the batch size, $y$ is the ground truth (a class from 0 to 3 representing each damage level), and $\hat{y}$ is the prediction. Ordinal cross-entropy loss differs from cross-entropy loss in the sense that it takes into account the distance between the ground truth and the predicted class (hence "ordinal"). Since the building damage classification problem involves different and increasing levels of damage from no damage to destruction, this function is useful to distinguish between different categories. To implement ordinal cross-entropy loss as the loss function, we treat it as generic multi-class classification and encode the classes no damage, minor damage, major damage, and destroyed as $[0,0,0],[1,0,0],[1$, $1,0]$, and $[1,1,1]$, respectively. [36]. The other aspects of the training process (optimizer, learning rate, number of epochs, etc.) remain the same. These improved models contribute to our understanding of what information leads to the most accurate prediction results for building damage assessment.

\subsection{Qualitative Interpretability}

In addition to the quantitative approaches achieved above, we also make progress in the study of model interpretability in a qualitative manner. One important question that one might ponder in the sphere of examining how, explicitly, models assess and classify building damage, is where the model is looking to make its decision. To address this question in a qualitative way, we create gradient class activation maps [37], or "heat maps," to represent which parts of an image (building crop) are most integral for the model's prediction. We do this after already training a model and saving its weights. For our purposes, we only perform this task on the baseline model, which takes in just the post-disaster image as input. 


\section{Results}

\subsection{Quantitative Results}

Table 1. Comparison of Validation Accuracy on 9 Different Models

\begin{tabular}{llll}
\hline \multicolumn{3}{c}{ Model Accuracy on Validation Set with Chosen Loss (100 epochs) } \\
\cline { 2 - 4 } \multicolumn{1}{c}{ Model Input } & \multicolumn{3}{c}{ Loss Function } \\
\hline Post-Disaster Image Only & $45.3 \%$ & $59.5 \%$ & $64.2 \%$ \\
\hline Pre-Disaster, Post-Disaster Images & $50.2 \%$ & $68.3 \%$ & $71.2 \%$ \\
\hline Pre-Disaster, Post-Disaster Images, Disaster Type & $49.7 \%$ & $72.7 \%$ & $74.6 \%$ \\
\hline
\end{tabular}

In Table 1, we present model accuracy on the validation set across nine different models, which are differentiated by three different input combinations and three different loss functions. The baseline model, which is trained with post-disaster data only and the cross-entropy loss function, has an accuracy of $59.5 \%$, as shown. It is important to note that all models were trained and validated on data that is evenly split between building crops of each class (no damage, minor damage, major damage, and destroyed), so a purely blind guessing model would achieve approximately $25 \%$ accuracy.

When the model is trained and validated on both pre-disaster and post-disaster building imagery as opposed to solely the post-disaster data, we see an $8.8 \%$ increase in accuracy on the validation set in comparison to the baseline model, while keeping the loss function constant. Adding the disaster type as a third type of input subsequently increases accuracy by another $4.4 \%$.

Reverting back to the baseline model, changing the loss function utilized to ordinal cross-entropy loss instead of simply cross-entropy loss, we see a $4.7 \%$ accuracy jump on the validation set. Sticking with ordinal cross-entropy loss, adding the pre-disaster image as a mode of input increases accuracy by another $7.0 \%$, while adding the disaster type as yet another mode of input increases accuracy by an additional $3.4 \%$.

\subsection{Qualitative Results}

In Figure 7, we present some qualitative results that we generated in the form of gradient class activation maps [37], as discussed earlier. Semantically, we can see that portions of the buildings that showed evidence of damage, such as cracks and debris, are depicted to have been the most useful to the model for classification.

\section{Discussion}

Much of our results conform to our hypotheses. Firstly, accuracy on the validation set improves when more modes of useful information are inputted into the model (accuracy generally increases moving down the rows of Table 1). This is reasonable because the more information that the model has to work with, the more accurate predictions it should make. A large part of our research was addressing which types of input aid the convolutional neural networks in making accurate predictions. From the results generated, it seems that having the aspect of change detection (when the pre-disaster image is concatenated with the post-disaster image and inputted) is useful, along with the type of disaster.

We also note that models using ordinal cross-entropy loss as their criterion for optimization perform the most accurately. This is also reasonable because, as previously mentioned, ordinal cross-entropy loss is most specifically applicable for a classification problem that involves an ordinal scale (in this case, the JDS), as opposed to categories with no intrinsic ordering. Mean squared error (MSE), not surprisingly, showed itself to be the least effective loss function to use for training. This is a 

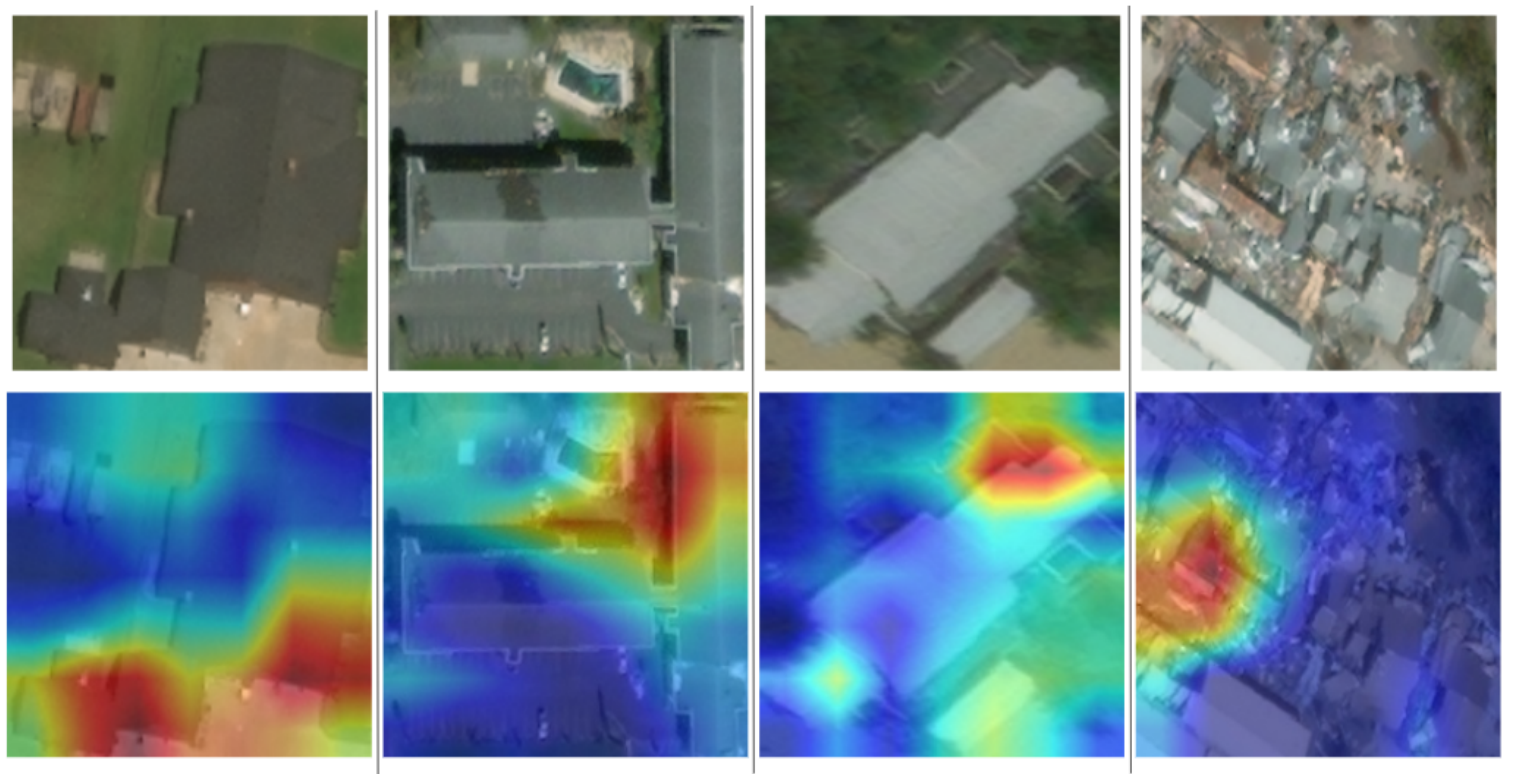

Figure 7. Gradient class activation maps depict which parts of the building crop lead the baseline model to predict a certain classification. On the top are the original images (crops) and on the bottom are the corresponding gradient class activation maps. The images included are only post-disaster images. From left to right: (1) A building with label "no damage," after flooding in the Midwestern United States, (2) A building with label "minor damage," after Hurricane Michael, (3) A building with label "major damage," after Hurricane Harvey, and (4) A building with label "destroyed," after Hurricane Michael.

justifiable because MSE is primarily used in regression problems, not classification problems. We find that cross-entropy loss models fall somewhere in between.

In summation, Table 1 shows that we were able to improve upon the baseline model by adding two more modes of input, as well as switching the loss function used to ordinal-cross entropy loss.

However, overall, we note that none of the accuracy numbers are necessarily optimal. This can be explained by the fact that the differences between categories, particularly between minor-damage and major-damage, are in many cases difficult to discern, for both humans and computers. This is certainly a challenge that comes with non-binary classification tasks with building damage, and it has been acknowledged by many, including Gupta et. al [10]. In addition, there is some noisy data in the dataset and cleaning it more thoroughly would most likely yield marginally more accurate model predictions.

Furthermore, the qualitative results generated in the form of gradient class activation maps display a more visual interpretation of what our baseline model is "seeing." These visuals can help in the study of other types of useful model inputs in the future.

These results, taken together, contribute to the research area of building damage detection by addressing the limited interpretability of current literature in regards to what types of information are most useful to building damage classification models as well as what loss functions are the best criterion.

\section{Conclusion}

The main insights that can be drawn from our work include using individualized building crops instead of semantic segmentation to train models, performing experiments with various combinations of model inputs and loss functions to explicitly examine their differences, and using overlaid heat maps to qualitatively analyze which aspects of a building are useful to derive predictions. Our work's main contribution to the field is presenting a novel, more interpretable and step-by-step, analysis of how to classify building damage most accurately and effectively in the event of a natural disaster. 
Practically, our work and others in the field advance methods for more robust emergency responses and more efficient allocation of resources, which saves lives and property. This research is especially important now, when climate change is ramping up the frequency and intensity of these devastating events.

Building on our work with improving interpretability, in the future, one could investigate the prediction performance of deep learning models with other types of input added, such as neighboring building damage levels. Experiments with other loss functions, if any, are also an aspect of future work in this area. Additionally, other ways to combine information such as pre-disaster and post-disaster images (instead of a simple concatenation like we did here) should yield interesting results. Moreover, it should be noted that the qualitative results we present are only from the baseline model. In the future, one could generate these types of gradient class activation maps for the models that take in both the pre-disaster and post-disaster images, or other models.

Funding: This research received no external funding

Acknowledgments: The author is very grateful for Ethan Weber, for his outstanding mentorship, and the Summer STEM Institute. This project would not have been possible without their support.

Conflicts of Interest: The authors declare no conflict of interest.

\section{References}

1. $\quad$ Ritchie, H.; Roser, M. Natural disasters. Our World in Data 2014.

2. Kadri, F.; Birregah, B.; Châtelet, E. The impact of natural disasters on critical infrastructures: A domino effect-based study. Journal of Homeland Security and Emergency Management 2014, 11, 217-241.

3. for Environmental Information, N.C. Billion-Dollar Weather and Climate Disasters: Overview.

4. Van Aalst, M.K. The impacts of climate change on the risk of natural disasters. Disasters 2006, 30, 5-18.

5. Hao, H.; Baireddy, S.; Bartusiak, E.R.; Konz, L.; LaTourette, K.; Gribbons, M.; Chan, M.; Comer, M.L.; Delp, E.J. An Attention-Based System for Damage Assessment Using Satellite Imagery. arXiv preprint arXiv:2004.06643 2020.

6. Gupta, R.; Shah, M. RescueNet: Joint Building Segmentation and Damage Assessment from Satellite Imagery. arXiv preprint arXiv:2004.07312 2020.

7. Xu, J.Z.; Lu, W.; Li, Z.; Khaitan, P.; Zaytseva, V. Building damage detection in satellite imagery using convolutional neural networks. arXiv preprint arXiv:1910.06444 2019.

8. Gueguen, L.; Hamid, R. Large-scale damage detection using satellite imagery. Proceedings of the IEEE Conference on Computer Vision and Pattern Recognition, 2015, pp. 1321-1328.

9. He, K.; Zhang, X.; Ren, S.; Sun, J. Deep residual learning for image recognition. Proceedings of the IEEE conference on computer vision and pattern recognition, 2016, pp. 770-778.

10. Gupta, R.; Hosfelt, R.; Sajeev, S.; Patel, N.; Goodman, B.; Doshi, J.; Heim, E.; Choset, H.; Gaston, M. Creating xBD: A Dataset for Assessing Building Damage from Satellite Imagery. The IEEE Conference on Computer Vision and Pattern Recognition (CVPR) Workshops, pp. 10-17.

11. Haralick, R.M.; Shanmugam, K.; Dinstein, I.H. Textural features for image classification. IEEE Transactions on systems, man, and cybernetics 1973, pp. 610-621.

12. Lu, D.; Weng, Q. A survey of image classification methods and techniques for improving classification performance. International journal of Remote sensing 2007, 28, 823-870.

13. Wang, J.; Yang, J.; Yu, K.; Lv, F.; Huang, T.; Gong, Y. Locality-constrained linear coding for image classification. 2010 IEEE computer society conference on computer vision and pattern recognition. IEEE, 2010, pp. 3360-3367.

14. Nath, S.S.; Mishra, G.; Kar, J.; Chakraborty, S.; Dey, N. A survey of image classification methods and techniques. 2014 International conference on control, instrumentation, communication and computational technologies (ICCICCT). IEEE, 2014, pp. 554-557.

15. Mikołajczyk, A.; Grochowski, M. Data augmentation for improving deep learning in image classification problem. 2018 international interdisciplinary PhD workshop (IIPhDW). IEEE, 2018, pp. 117-122. 
16. Wu, J.; Yu, Y.; Huang, C.; Yu, K. Deep multiple instance learning for image classification and auto-annotation. Proceedings of the IEEE conference on computer vision and pattern recognition, 2015, pp. 3460-3469.

17. Yang, X.; Ye, Y.; Li, X.; Lau, R.Y.; Zhang, X.; Huang, X. Hyperspectral image classification with deep learning models. IEEE Transactions on Geoscience and Remote Sensing 2018, 56, 5408-5423.

18. Deng, J.; Dong, W.; Socher, R.; Li, L.J.; Li, K.; Fei-Fei, L. Imagenet: A large-scale hierarchical image database. 2009 IEEE conference on computer vision and pattern recognition. Ieee, 2009, pp. 248-255.

19. Song, Z.; Chen, Q.; Huang, Z.; Hua, Y.; Yan, S. Contextualizing object detection and classification. CVPR 2011. IEEE, 2011, pp. 1585-1592.

20. Boutell, M.R.; Luo, J.; Shen, X.; Brown, C.M. Learning multi-label scene classification. Pattern recognition 2004, 37, 1757-1771.

21. Wang, H.; Kläser, A.; Schmid, C.; Liu, C.L. Action recognition by dense trajectories. CVPR 2011. IEEE, 2011, pp. 3169-3176.

22. Stoffle, R.W.; Halmo, D.B.; Wagner, T.W.; Luczkovich, J.J. Reefs from space: satellite imagery, marine ecology, and ethnography in the Dominican Republic. Human Ecology 1994, 22, 355-378.

23. Conway, B.; Browning, K.A. Weather forecasting by interactive analysis of radar and satellite imagery. Philosophical Transactions of the Royal Society of London. Series A, Mathematical and Physical Sciences 1988, 324, 299-315.

24. Rogers, D.J.; Randolph, S.E.; Snow, R.W.; Hay, S.I. Satellite imagery in the study and forecast of malaria. Nature 2002, 415, 710-715.

25. Novikov, G.; Trekin, A.; Potapov, G.; Ignatiev, V.; Burnaev, E. Satellite imagery analysis for operational damage assessment in emergency situations. International Conference on Business Information Systems. Springer, 2018, pp. 347-358.

26. Yang, C.; Everitt, J.H.; Du, Q.; Luo, B.; Chanussot, J. Using high-resolution airborne and satellite imagery to assess crop growth and yield variability for precision agriculture. Proceedings of the IEEE 2012, 101, 582-592.

27. Li, P.; Xu, H.; Song, B. A novel method for urban road damage detection using very high resolution satellite imagery and road map. Photogrammetric Engineering \& Remote Sensing 2011, 77, 1057-1066.

28. Asokan, A.; Anitha, J. Change detection techniques for remote sensing applications: a survey. Earth Science Informatics 2019, 12, 143-160.

29. Viña, A.; Echavarria, F.R.; Rundquist, D.C. Satellite change detection analysis of deforestation rates and patterns along the Colombia-Ecuador border. AMBIO: A Journal of the Human Environment 2004, 33, 118-125.

30. Hegazy, I.R.; Kaloop, M.R. Monitoring urban growth and land use change detection with GIS and remote sensing techniques in Daqahlia governorate Egypt. International Journal of Sustainable Built Environment 2015, 4, 117-124.

31. Weber, E.; Kané, H. Building Disaster Damage Assessment in Satellite Imagery with Multi-Temporal Fusion. AI for Earth Sciences Workshop at ICLR 2020.

32. Fujita, A.; Sakurada, K.; Imaizumi, T.; Ito, R.; Hikosaka, S.; Nakamura, R. Damage detection from aerial images via convolutional neural networks. 2017 Fifteenth IAPR International Conference on Machine Vision Applications (MVA). IEEE, 2017, pp. 5-8.

33. Chen, S.A.; Escay, A.; Haberland, C.; Schneider, T.; Staneva, V.; Choe, Y. Benchmark dataset for automatic damaged building detection from post-hurricane remotely sensed imagery. arXiv preprint arXiv:1812.05581 2018.

34. Foulser-Piggott, R.; Spence, R.; Saito, K.; Brown, D.; Eguchi, R. The use of remote sensing for post-earthquake damage assessment: lessons from recent events, and future prospects. Proceedings of the Fifthteenth World Conference on Earthquake Engineering, 2012, p. 10.

35. Paszke, A.; Gross, S.; Massa, F.; Lerer, A.; Bradbury, J.; Chanan, G.; Killeen, T.; Lin, Z.; Gimelshein, N.; Antiga, L.; others. Pytorch: An imperative style, high-performance deep learning library. Advances in neural information processing systems, 2019, pp. 8026-8037.

36. Cheng, J.; Wang, Z.; Pollastri, G. A neural network approach to ordinal regression. 2008 IEEE International Joint Conference on Neural Networks (IEEE World Congress on Computational Intelligence). IEEE, 2008, pp. 1279-1284. 
11 of 11

316 37. Selvaraju, R.R.; Cogswell, M.; Das, A.; Vedantam, R.; Parikh, D.; Batra, D. Grad-cam: Visual explanations 317 from deep networks via gradient-based localization. Proceedings of the IEEE international conference on $318 \quad$ computer vision, 2017, pp. 618-626. 